DESY 04-135

NIKHEF 2004-008

August 2004

\title{
The QCD Splitting Functions at Three Loops: Methods and Results*
}

\author{
S. Moch ${ }^{\mathrm{a}}$, J.A.M. Vermaseren ${ }^{\mathrm{b}}$ and A. Vogt ${ }^{\mathrm{b}}$ \\ aDeutsches Elektronensynchrotron DESY \\ Platanenallee 6, D-15735 Zeuthen, Germany \\ b NIKHEF Theory Group \\ Kruislaan 409, 1098 SJ Amsterdam, The Netherlands
}

We have computed the complete next-to-next-to-leading order (NNLO) contributions to the splitting functions governing the evolution of unpolarized parton densities in perturbative QCD. Our results agree with all partial results available in the literature. We illustrate the methods used for this calculation with some examples and display selected results to show the size of the NNLO corrections and their effect on the evolution.

\section{INTRODUCTION}

Parton distributions form indispensable ingredients for the analysis of all hard-scattering processes involving initial-state hadrons. The scaledependence of these distributions can be derived from first principles in terms of an expansion in powers of the strong coupling constant $\alpha_{\mathrm{s}}$. The corresponding $n$ th-order coefficients governing the evolution are referred to as the $n$-loop splitting functions. Including the terms up to order $\alpha_{\mathrm{s}}^{n+1}$ in the evolution of parton distributions, together with the corresponding results for the hard partonic cross sections of a given observable, one obtains the $\mathrm{N}^{n} \mathrm{LO}$ (leading-order, next-to-leading-order, next-to-next-to-leading-order, etc.) approximation of perturbative QCD.

The standard approximation for most important processes is presently the next-to-leading order, the corresponding one- and two-loop splitting functions being known for a long time. However, the NNLO corrections need to be included in order to arrive at quantitatively reliable predictions for hard processes at present and future high-energy colliders.

In a series of recent papers [123], we have published the complete unpolarized three-loop splitting functions. Here, we present some aspects of

\footnotetext{
${ }^{*}$ Presented by S.M. and J.A.M.V. at Loops and Legs in Quantum Field Theory, 25th - 30th April 2004, Zinnowitz (Germany).
}

the methods and selected results.

\section{METHODS}

The method of calculation employs the optical theorem, which relates the total cross section for a given process to the imaginary part of the forward Compton amplitude. We study deepinelastic scattering of a boson with Euclidean (offshell) momentum $Q$, off a parton with (on-shell) momentum $P$ and apply the operator product expansion. In the Bjorken limit, $Q^{2} \rightarrow \infty$ and $x=Q^{2} /(2 P \cdot Q)$ fixed, this yields a relation between matrix elements of parton operators of leading twist and Mellin moments of Feynman diagrams contributing to the forward Compton amplitude. Specifically, using dimensional regularization in $D=4-2 \epsilon$, we are able to compute the anomalous dimensions $\gamma$ of the parton operators, i.e. the integer- $N$ Mellin moments of splitting functions $P$,

$\gamma(N)=-\int_{0}^{1} d x x^{N-1} P(x)$

from the divergence in $\epsilon$ of the $N$-th Mellin moment of the corresponding Feynman diagrams.

In general, the (anti-)quark (anti-)quark splitting functions, constrained by charge conjugation invariance and flavour symmetry, are given for flavours $i, k$ by

$P_{\mathrm{q}_{i} \mathrm{q}_{k}}=P_{\overline{\mathrm{q}}_{i} \overline{\mathrm{q}}_{k}}=\delta_{i k} P_{\mathrm{qq}}^{\mathrm{v}}+P_{\mathrm{qq}}^{\mathrm{s}}$, 
$P_{\mathrm{q}_{i} \overline{\mathrm{q}}_{k}}=P_{\overline{\mathrm{q}}_{i} \mathrm{q}_{k}}=\delta_{i k} P_{\mathrm{q} \overline{\mathrm{q}}}^{\mathrm{v}}+P_{\mathrm{q} \overline{\mathrm{q}}}^{\mathrm{s}}$.

They can be composed into three independent types of non-singlet splitting functions,

$P_{\mathrm{ns}}^{ \pm}=P_{\mathrm{qq}}^{\mathrm{v}} \pm P_{\mathrm{q} \overline{\mathrm{q}}}^{\mathrm{v}}$,

and

$P_{\mathrm{ns}}^{\mathrm{v}}=P_{\mathrm{qq}}^{\mathrm{v}}-P_{\mathrm{q} \overline{\mathrm{q}}}^{\mathrm{v}}+n_{f}\left(P_{\mathrm{qq}}^{\mathrm{s}}-P_{\mathrm{q} \overline{\mathrm{q}}}^{\mathrm{s}}\right) \equiv P_{\mathrm{ns}}^{-}+P_{\mathrm{ns}}^{\mathrm{s}}$,

which govern the evolution of the quark flavour asymmetries $q_{\mathrm{ns}}^{ \pm}$and the valence distribution $q_{\mathrm{ns}}^{\mathrm{v}}$, respectively,

$\frac{d}{d \ln \mu_{f}^{2}} q_{\mathrm{ns}}^{i}=P_{\mathrm{ns}}^{i} \otimes q_{\mathrm{ns}}^{i}, \quad i= \pm, \mathrm{v}$.

The quark-quark singlet splitting function $P_{\mathrm{qq}}$ is expressed as

$P_{\mathrm{qq}}=P_{\mathrm{ns}}^{+}+n_{f}\left(P_{\mathrm{qq}}^{\mathrm{s}}+P_{\overline{\mathrm{qq}}}^{\mathrm{s}}\right) \equiv P_{\mathrm{ns}}^{+}+P_{\mathrm{ps}}$,

and gluon-quark and quark-gluon splitting functions are given by

$P_{\mathrm{qg}}=n_{f} P_{\mathrm{q}_{i} \mathrm{~g}}, \quad P_{\mathrm{gq}}=P_{\mathrm{gq}_{i}}$,

in terms of the flavour-independent splitting functions $P_{\mathrm{q}_{i} \mathrm{~g}}=P_{\overline{\mathrm{q}}_{i} \mathrm{~g}}$ and $P_{\mathrm{gq}_{i}}=P_{\mathrm{g}_{\bar{q}}}$. Thus, the singlet quark distribution $q_{\mathrm{s}}$ and the gluon distribution $g$ evolve according to

$\frac{d}{d \ln \mu_{f}^{2}}\left(\begin{array}{c}q_{\mathrm{s}} \\ g\end{array}\right)=\left(\begin{array}{cc}P_{\mathrm{qq}} & P_{\mathrm{qg}} \\ P_{\mathrm{gq}} & P_{\mathrm{gg}}\end{array}\right) \otimes\left(\begin{array}{c}q_{\mathrm{s}} \\ g\end{array}\right)$.

Eqs. (5) and (8) form the well-known $2 n_{f}-1$ scalar non-singlet and $2 \times 2$ singlet evolution equations, where $\otimes$ stands for the Mellin convolution.

In the present calculation, for the complete set of singlet and non-singlet splitting functions, we had to compute 18 one-loop Feynman diagrams at LO, 350 two-loop diagrams at NLO, and a total of 9607 three-loop diagrams at NNLO. All diagrams were generated automatically with the diagram generator QGRAF 4] and for all symbolic manipulations we used the latest version of FORM [5]. The NNLO calculation required significant enhancements of the capabilities of FORM [6].

In order to illustrate the details of the calculation, let us pick a particular Feynman diagram at three loops, which contributes to the forward Compton amplitude for the scattering of a photon off a quark.

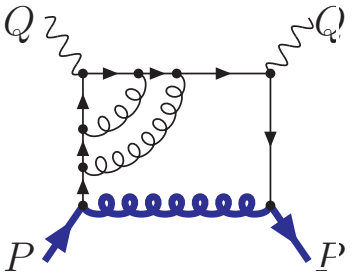

Here and below, the fat line indicates the flow of the quark momentum $P$ through the diagram.

In a first step, we need a classification of the topology of the loop integral. This is done in two steps. Any loop integral with external momenta $P$ and $Q$, we classify according to

1. the topology of the underlying "two-point function" if we nullify $P$.

2. the flow of the parton momentum $P$.

The first step yields at the top-level the topology types ladder, benz and non-planar, where we use the notation of Refs. [78. The second step distinguishes, within each topology, between so-called basic building blocks (BBB) which have a simple $P$-flow with one $P$-dependent propagator only, and so-called composite building blocks (CBB), which have a more complicated $P$-flow. At the top-level, there are $10 \mathrm{BBB}$ (3 ladder, 5 benz and 2 non-planar) and $32 \mathrm{CBB}$ (10 ladder, 16 benz and 6 non-planar). The smaller number of nonplanar topologies is due to symmetries. For illustration purposes, we represent the complete set of top-level BBB by pictograms as follows.

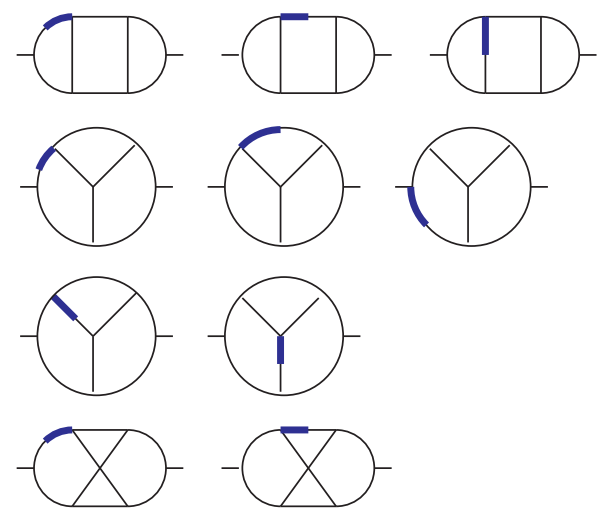

A quick inspection shows, that our example is a particular ladder type BBB, 
$--=\frac{(2 P \cdot Q)^{N}}{\left(Q^{2}\right)^{N+\alpha}} C_{N}$.

The equation indicates that we calculate the $N$-th Mellin moment of this diagram. This is precisely the dimensionless coefficient $C_{N}$ given on the right hand side. On the left hand side, the fat line in the pictogram represents the flow of the momentum $P$, in form of only one internal propagator containing both, a loop momentum, say $l_{1}$, and the external quark momentum $P$. Thus, on the left hand side the $N$-th term in a Taylor expansion generates the contribution to the $N$-th Mellin moment,

$\frac{1}{\left(P-l_{1}\right)^{2}}=\sum_{i} \frac{\left(2 P \cdot l_{1}\right)^{i}}{\left(l_{1}^{2}\right)^{i+1}} \longrightarrow \frac{\left(2 P \cdot l_{1}\right)^{N}}{\left(l_{1}^{2}\right)^{N}}$.

Thus far, the set-up is completely analogous to the calculation of the lowest six/seven (even or odd) integer- $N$ Mellin moments of the three-loop splitting functions 91011, where the MINCER program 78 was used as a tool to solve the integrals. As a new feature here we are dealing with symbolic $N$. This was tested before up to two loops [12. At three loops, it leads, for instance, to integrals of the type

$$
-n, k=\int \prod_{n}^{3} d^{D} l_{n} \frac{\left(2 P \cdot l_{1}\right)^{k}}{\left(l_{1}^{2}\right)^{n}} \frac{1}{l_{2}^{2} \ldots l_{8}^{2}},
$$

where the powers $n, k$ are symbolic.

However, we can switch to non-symbolic (fixed) positive powers $n, k$ and values of $N$ at any point of the derivations and calculations, after which the MiNCER program can be invoked to verify that the results are correct. From a practical point of view this is the most powerful feature of the Mellin-space approach, as it allows for extremely efficient checks.

Having classified the integrals, we actually need to solve them, too. To this end, let us start the discussion with the most general form of a threeloop integral in one of the top-level topologies ladder, benz or non-planar,

$$
\begin{aligned}
& I(N, \vec{\mu}, \vec{\nu}, \vec{\kappa})=\int \prod_{n=1}^{3} d^{D} l_{n} \\
& \frac{\left(2 P \cdot l_{1}\right)^{\kappa_{1}} \ldots\left(2 P \cdot l_{8}\right)^{\kappa_{8}}\left(2 l_{9} \cdot l_{10}\right)^{\kappa_{9}}}{\left(l_{1}^{2}\right)^{\mu_{1}}\left(\left(P-l_{1}\right)^{2}\right)^{\nu_{1}} \ldots\left(l_{8}^{2}\right)^{\mu_{8}}\left(\left(P-l_{8}\right)^{2}\right)^{\nu_{8}}} .
\end{aligned}
$$

Here, $\vec{\mu}=\mu_{1}, \ldots, \mu_{8}, \vec{\nu}=\nu_{1}, \ldots, \nu_{8}$ and $\vec{\kappa}=$ $\kappa_{1}, \ldots, \kappa_{9}$ are symbolic parameters. Furthermore, the $l_{i}$ are for $i=1,2,3$ independent loop momenta. For $i=4, \ldots, 8$ they can be expressed by the former and the external momentum $Q$, the precise relations depending on the topology. The momenta $l_{9}, l_{10}$ denote an irreducible scalar product between the momenta $Q$ and/or $l_{i}, i=1, \ldots, 8$, again the precise relation being topology depended.

Applying relations based on integration by parts [1314 15 16, scaling equations, form-factor analysis 17 and some equations 2 that fall in a special category because they involve higher twist and a careful study of the parton-momentum limit $P \cdot P \rightarrow 0$, we arrive at a system of linear equations for a given integral $I(N, \vec{\mu}, \vec{\nu}, \vec{\kappa})$ under consideration.

Solving this linear system amounts to finding a scheme in which $I(N, \vec{\mu}, \vec{\nu}, \vec{\kappa})$ is mapped to a set of master integrals and integrals of simpler topologies. The general strategy applies two basic rules of mapping,

1. non-planar $\longrightarrow$ benz $\longrightarrow$ ladder.

\section{2. $\mathrm{CBB} \longrightarrow \mathrm{BBB}$}

Here, the first rule is understood to hold for common sub-topologies. For instance, the non-planar topology contains common sub-topologies with the benz and the ladder topology 78 .

In an operator approach, this can be realized by diagonalizing the linear system for symbolic indices. One thus obtains lowering operators for individual $\nu_{i}$ or $\mu_{i}$, as well as recursion relations in the Mellin moment $N$. The latter constitute difference equations, which may generally be written as

$$
\begin{aligned}
& a_{0}(N) I(N)+a_{1}(N) I(N-1) \\
& \quad+\ldots+a_{m}(N) I(N-m)=G(N) .
\end{aligned}
$$

To illustrate the latter, let us give an extremely simple example for a single-step difference equation in $N$, which occurs in the reduction of a particular type of $\mathrm{CBB}$, 


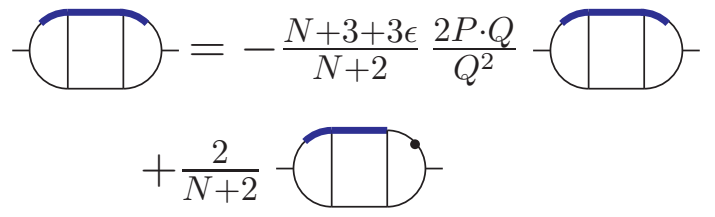

In the pictogram, all lines denote propagators of unit power, except the one with a blob, which has to be taken to second power. Again, it is understood that the equation holds for the $N$-th Mellin moment of the diagrams and the fat lines indicate propagators with the momentum $P$.

Employing the notation of Eq. (11), we can write the single-step difference equations as

$I(N)=-\frac{N+3+3 \epsilon}{N+2} I(N-1)+\frac{2}{N+2} G(N)$.

As a remark on the side, imagine for a moment, the function $G(N)$ on right hand side would be multiplied by an additional factor $\epsilon^{-1}$. If present, such a so-called spurious pole in $\epsilon$ would make Eq. (12) useless. It would ruin the accuracy of the expansion when working only a to given cutoff in powers of $\epsilon$. (We do so both for reasons of economy and because we cannot evaluate some integrals easily beyond certain powers in $\epsilon$.) Thus, spurious poles have to be avoided and one of the greatest difficulties in deriving reduction schemes is to indeed avoid them.

Eq. (12) can be solved in closed form,

$$
\begin{aligned}
I(N) & =(-1)^{N} \frac{\prod_{j=1}^{N}(j+3+3 \epsilon)}{\prod_{j=1}^{N}(j+2)} I(0) \\
& +(-1)^{N} \sum_{i=1}^{N}(-1)^{j} \frac{\prod_{j=i+1}^{N}(j+3+3 \epsilon)}{\prod_{j=i}^{N}(j+2)} G(i) .
\end{aligned}
$$

Eq. (13) is an example for the occurrence of nested sums in the calculation. Its solution requires as input the boundary value $I(0)$, which can be obtained with the Mincer program. The inhomogeneous term $G(N)$ is assumed to be already known. It has to be calculated by similar means, i.e. reductions and recursions. The successive way of solving difference equations induces a strict hierarchy for all topology classes in the reduction scheme.

As a matter of fact, Eq. (13) is a special case of a general recursion which was derived for $I(N, \vec{\mu}, \vec{\nu}, \vec{\kappa})$ with symbolic indices, like most other recursions as well. As such, they allow for an efficient implementation in FORM resulting in a largely automatic build-up of nested sums.

The solution of nested sums as in Eq. (13) results in harmonic sums 1819202122 which are recursively defined as

$S_{ \pm m_{1}, \ldots, m_{k}}(N)=\sum_{i=1}^{N} \frac{( \pm 1)^{i}}{i^{m_{1}}} S_{m_{2}, \ldots, m_{k}}(i)$.

In particular, we use four main algorithms for harmonic sums, which rely on the underlying algebra 2324]. The algorithms express products or sums of nested sums again in the basis Eq.(14) of harmonic sums. Specifically, they act on products,

$S_{m_{1}, \ldots, m_{k}}(N) S_{n_{1}, \ldots, n_{l}}(N)$,

sums involving $j$ and $N-j$

$$
\begin{aligned}
& \sum_{j=1}^{N-1} \frac{1}{j^{m_{1}}} S_{m_{2}, \ldots, m_{k}}(j) \frac{1}{(N-j)^{n_{1}}} \\
& \times S_{n_{2}, \ldots, n_{l}}(N-j),
\end{aligned}
$$

conjugations

$$
-\sum_{j=1}^{N}\left(\begin{array}{c}
N \\
j
\end{array}\right)(-1)^{j} \frac{1}{j^{m_{1}}} S_{m_{2}, \ldots, m_{k}}(j),
$$

and on sums involving binomials, $j$ and $N-j$,

$$
\begin{gathered}
-\sum_{j=1}^{N-1}\left(\begin{array}{c}
N \\
j
\end{array}\right)(-1)^{j} \frac{1}{j^{m_{1}}} S_{m_{2}, \ldots, m_{k}}(j) \\
\times \frac{1}{(N-j)^{n_{1}}} S_{n_{2}, \ldots, n_{l}}(N-j) .
\end{gathered}
$$

The solution to Eq. 13) up to order $\epsilon^{-1}$ as required for the calculation of the splitting functions can be obtained,

$$
\begin{gathered}
I(N)=(-1)^{N} \frac{1}{\epsilon^{2}}\left(\frac{4}{3} \frac{S_{1}(N+1)}{N+1}+\frac{8}{3} \frac{S_{1}(N+1)}{(N+1)^{2}}\right. \\
+\frac{4}{3} \frac{S_{1}(N+2)}{N+2}+\frac{4}{3} \frac{S_{1}(N+2)}{(N+2)^{2}}+\frac{4}{3} S_{1}(N) \\
+\frac{2}{3} S_{1,2}(N)+\frac{2}{3} \frac{S_{2}(N+1)}{N+1}+\frac{2}{3} \frac{S_{2}(N+2)}{N+2}
\end{gathered}
$$




$$
\begin{aligned}
& -2 S_{2}(N)-\frac{4}{3} N S_{2}(N)+4 S_{2,1}(N) \\
& +\frac{4}{3} N S_{2,1}(N)-6 S_{3}(N)-2 N S_{3}(N) \\
& -\frac{8}{3} \frac{1}{(N+1)^{2}}-4 \frac{1}{(N+1)^{3}}-\frac{4}{3} \frac{1}{(N+2)^{2}} \\
& \left.-2 \frac{1}{(N+2)^{3}}\right)+(-1)^{N} \frac{1}{\epsilon}\left(-16 \frac{S_{1}(N+1)}{N+1}\right. \\
& -\frac{88}{3} \frac{S_{1}(N+1)}{(N+1)^{2}}-\frac{20}{3} \frac{S_{1}(N+1)}{(N+1)^{3}}-16 \frac{S_{1}(N+2)}{N+2} \\
& -\frac{44}{3} \frac{S_{1}(N+2)}{(N+2)^{2}}-\frac{10}{3} \frac{S_{1}(N+2)}{(N+2)^{3}}-20 S_{1}(N) \\
& +\frac{8}{3} \frac{S_{1,1}(N+1)}{N+1}+\frac{8}{3} \frac{S_{1,1}(N+1)}{(N+1)^{2}}+\frac{8}{3} \frac{S_{1,1}(N+2)}{N+2} \\
& +\frac{8}{3} S_{1,1}(N)+\frac{10}{3} S_{1,1,2}(N)+\frac{10}{3} \frac{S_{1,2}(N+1)}{N+1} \\
& +\frac{10}{3} \frac{S_{1,2}(N+2)}{N+2}-16 S_{1,2}(N)-4 N S_{1,2}(N) \\
& +14 S_{1,2,1}(N)+4 N S_{1,2,1}(N)-24 S_{1,3}(N) \\
& -6 N S_{1,3}(N)-\frac{58}{3} \frac{S_{2}(N+1)}{N+1}-\frac{40}{3} \frac{S_{2}(N+1)}{(N+1)^{2}} \\
& -\frac{46}{3} \frac{S_{2}(N+2)}{N+2}-6 \frac{S_{2}(N+2)}{(N+2)^{2}}+\frac{56}{3} S_{2}(N) \\
& +20 N S_{2}(N)+10 \frac{S_{2,1}(N+1)}{N+1}+6 \frac{S_{2,1}(N+2)}{N+2} \\
& -\frac{134}{3} S_{2,1}(N)-\frac{56}{3} N S_{2,1}(N)+\frac{16}{3} S_{2,1,1}(N) \\
& +\frac{8}{3} N S_{2,1,1}(N)-\frac{62}{3} S_{2,2}(N)-\frac{22}{3} N S_{2,2}(N) \\
& -18 \frac{S_{3}(N+1)}{N+1}-12 \frac{S_{3}(N+2)}{N+2}+76 S_{3}(N) \\
& +\frac{100}{3} N S_{3}(N)-10 S_{3,1}(N)-\frac{10}{3} N S_{3,1}(N) \\
& +36 S_{4}(N)+12 N S_{4}(N)+32 \frac{1}{(N+1)^{2}} \\
& +\frac{164}{3} \frac{1}{(N+1)^{3}}+24 \frac{1}{(N+1)^{4}}+16 \frac{1}{(N+2)^{2}} \\
& \left.+\frac{82}{3} \frac{1}{(N+2)^{3}}+12 \frac{1}{(N+2)^{4}}\right) \text {. }
\end{aligned}
$$

The result in Eq. (19) is neither short nor inexpensive to calculate. Moreover, each integral is typically used many times. Therefore, to save computer time and disk space, we have tabulated large numbers of $\mathrm{CBB}$ and $\mathrm{BBB}$ integrals. Only this tabulation rendered the calculation feasible with current computing resources. For the complete project we have collected table-bases with more than 100.000 integrals and a total size of tables of more than 3 GBytes.

Subsequently, the database of integrals was used for the calculation of all Feynman diagrams yielding the unrenormalized results in Mellinspace in terms of the invariants determined by the colour group 25, harmonic sums and the values $\zeta_{3}, \zeta_{4}, \zeta_{5}$ of the Riemann $\zeta$-function. In physics results the terms with $\zeta_{4}$ cancel in $N$-space. The renormalization was carried out in the $\overline{\mathrm{MS}}$-scheme [2627] and the procedure is again the same as for the fixed-moment calculations 91011.

\section{RESULTS}

Now we present the anomalous dimensions $\gamma\left(\alpha_{\mathrm{s}}, N\right)$ up to the third order in the running coupling constant $\alpha_{\mathrm{s}}$, the expansion coefficients being normalized as

$\gamma\left(\alpha_{\mathrm{s}}, N\right)=\sum_{n=0}\left(\frac{\alpha_{\mathrm{s}}}{4 \pi}\right)^{n+1} \gamma^{(n)}(N)$.

Our analytical results have been presented in Refs. 123 and are too long to be reproduced here. We agree with all partial results available in the literature, in particular we reproduce the lowest six even integer singlet and seven even/odd integer non-singlet moments ${ }^{2}$ computed before 91011.

The numerical results for the singlet anomalous dimension, i.e. the Mellin transforms of the matrix entries in Eq. (8) are illustrated in Fig. 10 In the top row of Fig. 1] we show the perturbative expansion of the diagonal anomalous dimensions $\gamma_{\mathrm{qq}}(N)$ and $\gamma_{\mathrm{gg}}(N)$ for four flavours at $\alpha_{\mathrm{s}}=0.2$. The pure-singlet (ps) contribution to $\gamma_{\mathrm{qq}}$ as defined in Eq. (6) is displayed separately. The bottom row of Fig. 1 shows the off-diagonal anomalous dimensions $\gamma_{\mathrm{qg}}(N)$ and $\gamma_{\mathrm{gq}}(N)$. For all cases, the NNLO corrections are significantly smaller than the NLO contributions and amount

${ }^{2}$ The moment $N=16$ of $\gamma_{\mathrm{ns}}^{(2)+}$ has recently been computed as an additional check [28]. 

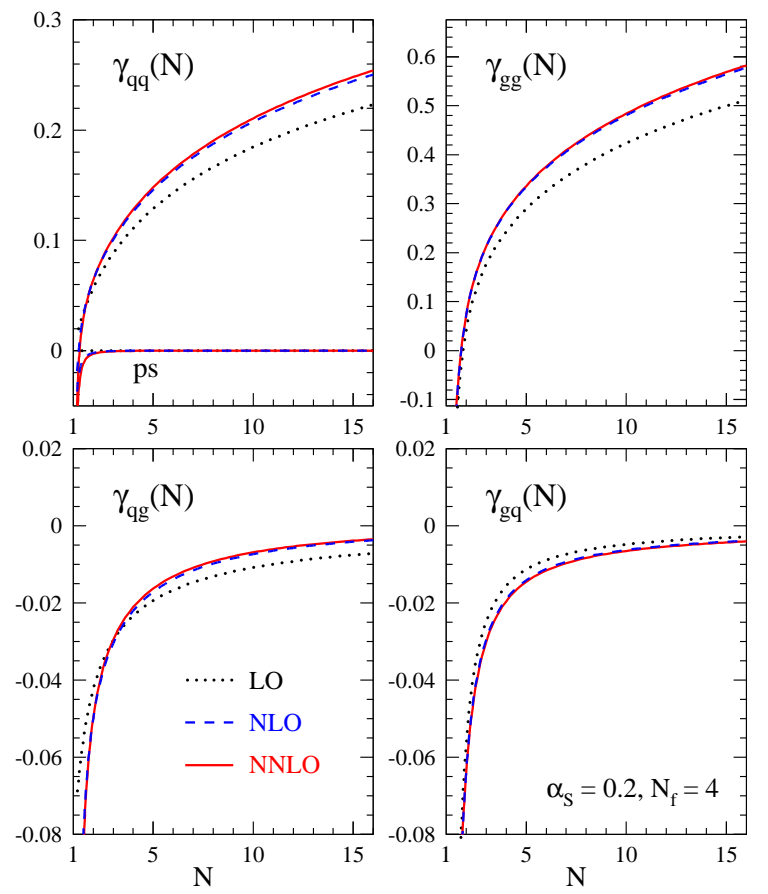

Figure 1. The perturbative expansion of the singlet anomalous dimensions $\gamma\left(\alpha_{s}, N\right)$.

to less than $2 \%$ and $1 \%$ for the large diagonal quantities $\gamma_{\mathrm{qq}}$ and $\gamma_{\mathrm{gg}}$, respectively, for $N>2$.

In Bjorken $x$-space, the $\mathrm{N}^{n} \mathrm{LO}$ splitting functions $P^{(n)}(x)$ in

$P\left(\alpha_{\mathrm{s}}, x\right)=\sum_{n=0}\left(\frac{\alpha_{\mathrm{s}}}{4 \pi}\right)^{n+1} P^{(n)}(x)$,

can be obtained from Eq. (1) and expressed in terms of harmonic polylogarithms 293031 by means of an inverse Mellin transformation. This is a completely algebraic procedure based on the fact that harmonic sums occur as coefficients of the Taylor expansion of harmonic polylogarithms.

Again, the analytical results have been given in Refs. 123. They agree with the available resummation predictions 32 33 34 35 for the leading small- $x$ logarithms, and those for large- $n_{f}$ results 36 37. In addition, we have also provided easy-to-use accurate parameterizations.

Our results respect the supersymmetric relation between all four singlet splitting functions for

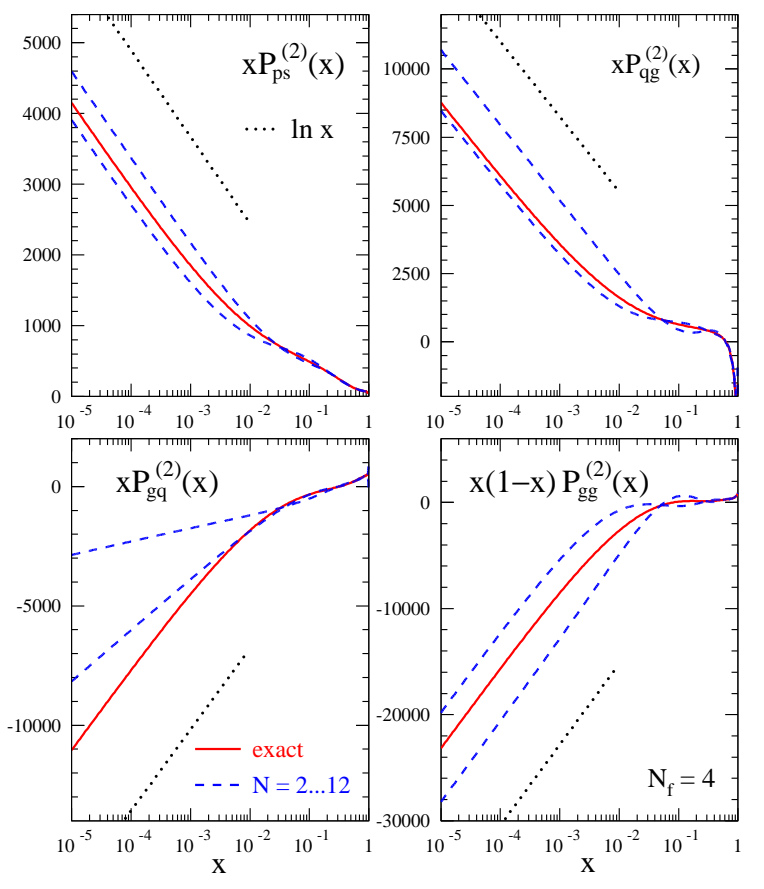

Figure 2. The three-loop singlet splitting functions $P_{\mathrm{ab}}^{(2)}$ with the leading small- $x$ terms (dotted) and the fixed-moment estimates (dashed).

$C_{A}=C_{F}=n_{f}$ to the extend expected for the $\overline{\mathrm{MS}}$ scheme. At large $x$ we agree with Refs. 3839 and determine for $P_{\mathrm{ns}}^{ \pm, \mathrm{v}}, P_{\mathrm{qq}}$ and $P_{\mathrm{gg}}$ the coefficients of the leading $1 /(1-x)+$ terms. We find that the coefficients of the leading integrable term $\ln (1-x)$ at order $n=2,3$ are proportional to the coefficient of the +-distribution $1 /(1-x)_{+}$ at order $n-1$, a result that seems to point to a yet unexplored structure. Furthermore, we verify the expected simple relation between the leading $1 /(1-x)_{+}$terms of $P_{\mathrm{qq}}$ and $P_{\mathrm{gg}}$.

In Fig. 2] we plot the three-loop singlet splitting functions of Eq. (8) in the small- $x$ region, where the leading small- $x$ behaviour is of the type $x^{-1} \ln x$. This agrees with the prediction of the leading logarithmic BFKL equation 40 4142. In the top row of Fig. 2 we give the three-loop splitting functions $P_{\mathrm{ps}}^{(2)}$ (pure-singlet quark-quark) and $P_{\mathrm{qg}}^{(2)}$ (gluon-quark) for four flavours, multiplied by $x$ for display purposes. Also shown are 


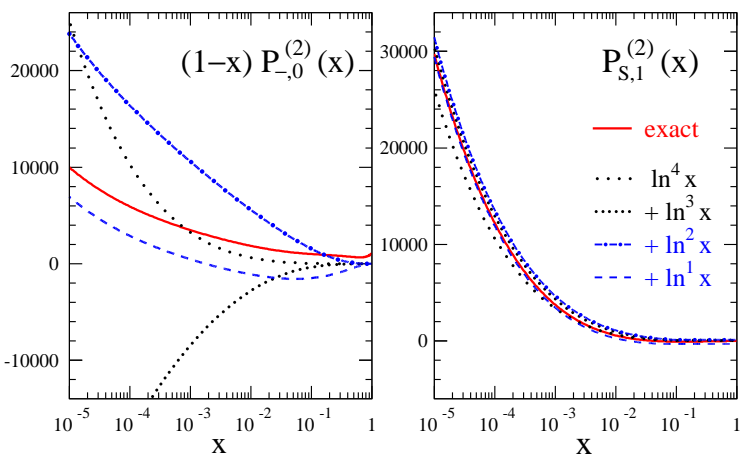

Figure 3. The non-singlet three-loop splitting functions $P_{\mathrm{ns}}^{-}\left(n_{f}\right.$-independent part) and $P_{\mathrm{ns}}^{s} / n_{f}$.

the uncertainty band derived in Ref. 43] using the lowest six even-integer moments [1011] and the leading small- $x$ terms 32 . In the bottom row of Fig. 2 we show the three-loop splitting functions $P_{\mathrm{gq}}^{(2)}$ (quark-gluon) and $P_{\mathrm{gg}}^{(2)}$ (gluon-gluon). For $P_{\mathrm{gq}}^{(2)}$, the leading small- $x$ contribution was unknown before the present calculation, for $P_{\mathrm{gg}}^{(2)}$ the leading small- $x$ term has been first obtained in Ref. 33. $P_{\mathrm{gg}}^{(2)}$ as a diagonal quantity has been additionally multiplied by $(1-x)$. As illustrated in Fig. 2 the leading small- $x$ terms alone do not provide good approximations of the full results at experimentally relevant small values of $x$. At $x=10^{-4}$, for example, they exceed the exact values of $P_{\mathrm{ab}}^{(2)}(x)$ by factors between 1.6 and 2.0 for $n_{f}=4$.

In Fig. B we display the three-loop non-singlet splitting functions $P_{-}^{(2)}(x)$ and $P_{\mathrm{ns}}^{(2) \mathrm{s}}$. On the left hand side of Fig. 3 the $n_{f}$-independent threeloop contribution $P_{-, 0}^{(2)}(x)$ to the splitting function $P_{\mathrm{ns}}^{-}(x)$, multiplied by $(1-x)$ for display purposes is shown. Also shown is a comparison of our exact result to the small- $x$ expansion in powers of $\ln x$. Here, the leading small- $x$ terms of the type $\ln ^{4} x$ were known before for $P_{\mathrm{ns}}^{(2) \pm}(x)$ 3435. As can be seen from Fig. 3 on the left, the coefficients of $\ln ^{k} x$ increase sharply with decreasing power $k$. Including all logarithmically enhanced terms, one still underestimates the complete result by a factor as large as 2.0 for $P_{\mathrm{ns}}^{(2)-}$ at $x=10^{-4}$.
The three-loop contribution $P_{\mathrm{ns}}^{(2) \mathrm{s}}$ exhibits a new colour structure $d^{a b c} d_{a b c} / n_{c}$ which appears for the first time at three loops. It is due to Feynman graphs of the following type, involving axial currents (the quarks couple to $W$-bosons),

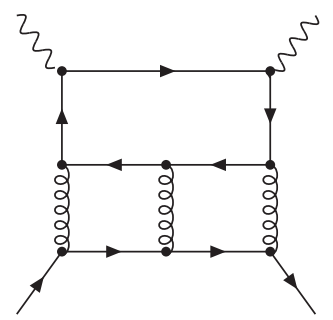

Recall that at one and two loops $P_{\mathrm{ns}}^{(0) \mathrm{s}}$ and $P_{\mathrm{ns}}^{(1) \mathrm{s}}$ both vanish. $P_{\mathrm{ns}}^{(2) \mathrm{s}}$ is displayed in Fig. [3 on the right. Quiet unexpectedly, $P_{\mathrm{ns}}^{(2) \mathrm{s}}$ also behaves like $\ln ^{4} x$ for $x \rightarrow 0$, and here the leading small- $x$ terms do indeed provide a reasonable approximation. In fact, this function dominates the small- $x$ behaviour of the non-singlet splitting functions, for $n_{f}=4$ being, for example, about 7 times larger than $P_{\mathrm{ns}}^{(2) \pm}(x)$ at $x=10^{-4}$.

Let us next illustrate the numerical effect of the three-loop splitting functions $P_{\mathrm{ab}}^{(2)}(x)$ on the evolution of the singlet-quark and gluon distributions $q_{\mathrm{s}}\left(x, \mu_{f}^{2}\right)$ and $g\left(x, \mu_{f}^{2}\right)$. For all figures we choose a reference scale $\mu_{f}^{2}=\mu_{0}^{2} \simeq 30 \mathrm{GeV}^{2}$ - a scale relevant, for example, for deep-inelastic scattering both at fixed-target experiments and the $e p$ collider HERA - and employ the sufficiently realistic model distributions

$$
\begin{aligned}
& x q_{\mathrm{s}}\left(x, \mu_{0}^{2}\right)= \\
& \quad 0.6 x^{-0.3}(1-x)^{3.5}\left(1+5.0 x^{0.8}\right), \\
& x g\left(x, \mu_{0}^{2}\right)= \\
& \quad 1.6 x^{-0.3}(1-x)^{4.5}\left(1-0.6 x^{0.3}\right),
\end{aligned}
$$

irrespective of the order of the expansion to facilitate the comparison of the LO, NLO and NNLO contributions to the splitting functions.

In Fig. 团 we show in the top row the perturbative expansion of the scale derivative $\dot{q}_{\mathrm{s}} \equiv$ $d \ln q_{\mathrm{s}} / d \ln \mu_{f}^{2}$ of the singlet quark distribution, i.e. the top row of Eq. (8), at $\mu_{f}^{2}=\mu_{0}^{2}$ for four flavours, $\alpha_{\mathrm{s}}=0.2$, and the initial conditions specified in Eqs. (22), (23). The bottom row of 

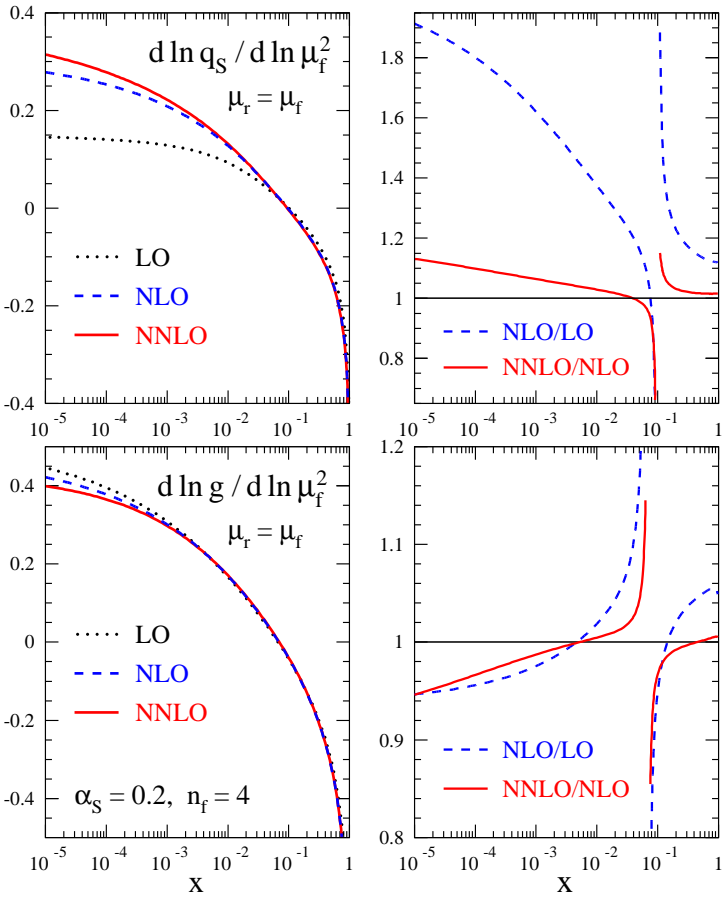

Figure 4. The perturbative expansion of the scale derivatives (8) of the singlet distributions (22), 23].

Fig. 4 shows the same for the gluon distribution $g$, i.e. for the bottom row of Eq. (8). The spikes close to $x=0.1$ in the right parts of both figures are due to zeros of the LO and NLO predictions and do not represent large corrections.

The NNLO corrections are small at large $x$ with respect to both the total derivative and the NLO contributions. At small- $x$ the NLO contributions are very large for the quark evolution. Consequently the total NNLO corrections, while reaching $10 \%$ at $x=10^{-4}$, remain smaller than the NLO results by a factor of eight or more over the full $x$-range.

In Fig. 5]we show the perturbative expansion of the logarithmic scale derivative $d \ln q_{\mathrm{ns}}^{-} / d \ln \mu_{f}^{2}$ for a characteristic non-singlet quark distribution

$x q_{\mathrm{ns}}^{i}=x^{0.5}(1-x)^{3}, \quad i= \pm, \mathrm{v}$,

at the standard scale $\mu_{r}=\mu_{f}$. In addition, on the right hand side of Fig. 5 , the non-singlet quark

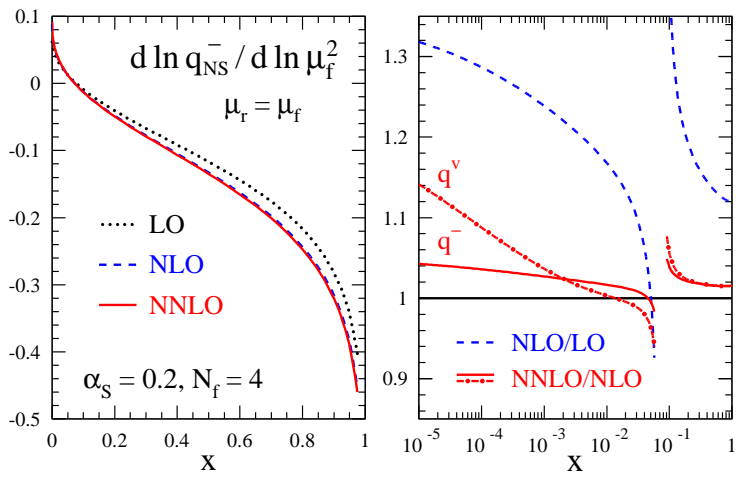

Figure 5. The perturbative expansion of the scale derivative (5) of the non-singlet distributions $q_{-}$ and $q_{\mathrm{v}}$ for the input (24).

distribution $q_{\mathrm{ns}}^{\mathrm{v}}$ is also displayed.

Finally, we turn to the stability of the perturbative expansions under variations of the renormalization scale $\mu_{r}$. For $\mu_{r} \neq \mu_{f}$ the expansion of the splitting functions in Eq. (21) is, using the abbreviation $a_{\mathrm{s}} \equiv \alpha_{\mathrm{s}} /(4 \pi)$, replaced by

$$
\begin{gathered}
P_{\mathrm{ab}}\left(\mu_{f}, \mu_{r}\right)=a_{\mathrm{s}}\left(\mu_{r}^{2}\right) P_{\mathrm{ab}}^{(0)} \\
+a_{\mathrm{s}}^{2}\left(\mu_{r}^{2}\right)\left(P_{\mathrm{ab}}^{(1)}-\beta_{0} P_{\mathrm{ab}}^{(0)} \ln \frac{\mu_{f}^{2}}{\mu_{r}^{2}}\right) \\
+a_{\mathrm{s}}^{3}\left(\mu_{r}^{2}\right)\left(P_{\mathrm{ab}}^{(2)}-\left\{\beta_{1} P_{\mathrm{ab}}^{(0)}+2 \beta_{0} P_{\mathrm{ab}}^{(1)}\right\} \ln \frac{\mu_{f}^{2}}{\mu_{r}^{2}}\right. \\
\left.+\beta_{0}^{2} P_{\mathrm{ab}}^{(0)} \ln ^{2} \frac{\mu_{f}^{2}}{\mu_{r}^{2}}\right)+\ldots,
\end{gathered}
$$

where $\beta_{k}$ represent the $\overline{\mathrm{MS}}$ expansion coefficients of the $\beta$-function of QCD 44454647 .

In Fig. 6] the relative scale uncertainties of the average results is plotted, which is conventionally estimated by

$\Delta \dot{f} \equiv \frac{\max \left[\dot{f}\left(x, \mu_{r}^{2}\right)\right]-\min \left[\dot{f}\left(x, \mu_{r}^{2}\right)\right]}{2 \mid \text { average }\left[\dot{f}\left(x, \mu_{r}^{2}\right)\right] \mid}$,

where the scale $\mu_{r}^{2}$ varies $\mu_{r}^{2} \in\left[\frac{1}{4} \mu_{f}^{2}, 4 \mu_{f}^{2}\right]$.

The top row of Fig. [6 shows the renormalization scale uncertainty of the NLO and NNLO predictions for the scale derivative of $q_{\mathrm{ns}}^{i}, i= \pm, \mathrm{v}$, as obtained from the quantity $\Delta \dot{q}_{\text {ns }}^{i}$ defined in 

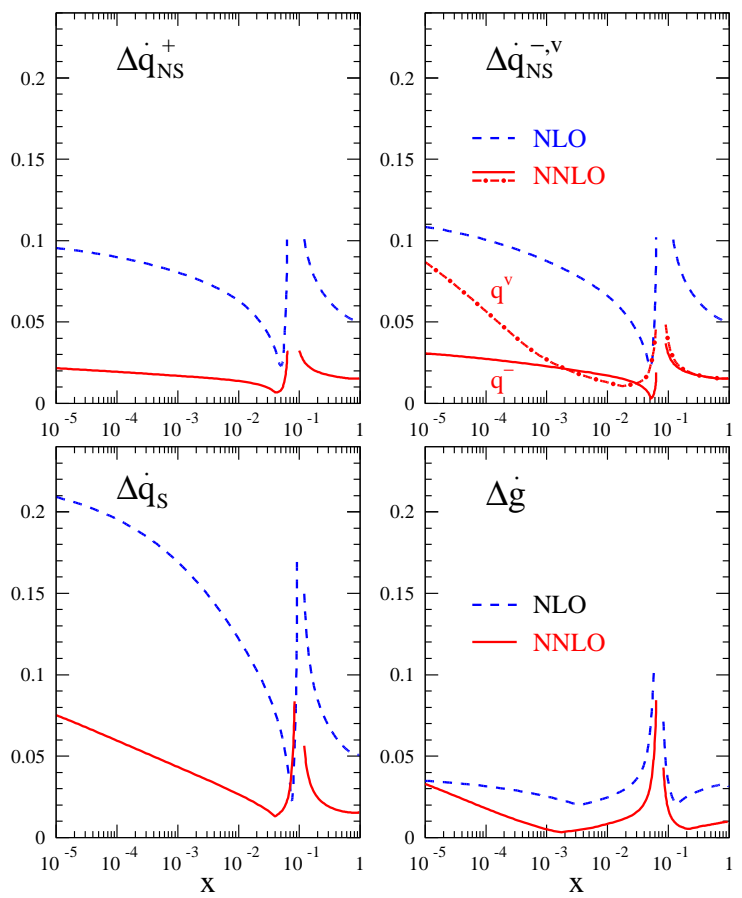

Figure 6. The relative scale uncertainty $\Delta \dot{f}$ (26) for all non-singlet and singlet cases.

Eq. 267. The bottom row displays the same, but for the singlet-quark distributions $q_{\mathrm{s}}$ and the gluon distribution $g$. For all quark distribution, these uncertainty estimates amount to $2 \%$ or less at $x>10^{-2}$ (for the gluon distribution $1 \%$ at $\left.x>3 \cdot 10^{-4}\right)$, an improvement by more than a factor of three with respect to the corresponding NLO results.

In general, for $x \gtrsim 10^{-3}$ the perturbative expansion for the scale derivatives $\dot{f} \equiv$ $d \ln f\left(x, \mu_{f}^{2}\right) / d \ln \mu_{f}^{2}, f=q_{\mathrm{ns}}^{i}, q_{\mathrm{s}}, g$ appears to be very well convergent and suggests a residual higher-order uncertainty of about $1 \%$ or less at $\alpha_{\mathrm{s}} \lesssim 0.2$. Consequently the perturbative evolution can be safely extended to considerably larger values of $\alpha_{\mathrm{s}}$, hence lower scales, in this range of $x$. Larger corrections have to be expected at small $x$, but the results of the small- $x$ resummation alone will not help here. Further progress at small $x$ would require at least a four-loop generalization of the fixed- $N$ calculations 91011 and of the $x$-space approximations 43 linking them to the small- $x$ limits. In addition, one should also keep in mind that at fourth order the new colour structure $d^{a b c} d_{a b c} / n_{c}$ also will appear in singlet splitting functions.

\section{CONCLUSION}

We have calculated the complete third-order contributions to the splitting functions governing the evolution of unpolarized parton distribution in perturbative QCD.

The calculation is performed in Mellin- $N$ space and follows the previous fixed-integer $N$ computations 91011. The extension to the complete analytical $N$-dependence is the crucial new feature. It required the set-up of an elaborate reduction scheme for the corresponding loop integrals, an improved understanding of the mathematics of harmonic sums, difference equations and harmonic polylogarithms, and finally the implementation of corresponding tools, together with other new features [6], in the symbolic manipulation program FORM [5].

Furthermore, by keeping terms of order $\varepsilon^{0}$ in dimensional regularization throughout the calculation, we have also obtained the third-order coefficient functions for the structure functions $F_{2}$ and $F_{L}$ in electromagnetic and for $F_{3}$ in chargedcurrent deep-inelastic scattering [48. Additionally, the present method can be used to generalize our fixed- $N$ three-loop calculation of the photon structure 49] to all $N$ and it should also be possible to obtain the polarized NNLO splitting functions in this manner.

The results for the three-loop splitting functions have been presented in both Mellin- $N$ and Bjorken- $x$ space in Refs. 1123 and agree with all partial results available in the literature. We have investigated the numerical impact of the threeloop (NNLO) contributions on the evolution of the parton distributions. The perturbative expansion appears to be very well convergent except for very small $x$ and shows good stability under variation of the scales. Thus, with the results presented, the precision of the perturbative predictions for parton evolution has been greatly improvement. 


\section{REFERENCES}

1. S. Moch, J. Vermaseren and A. Vogt, Nucl. Phys. B646 (2002) 181, hep-ph/0209100

2. S. Moch, J. Vermaseren and A. Vogt, Nucl. Phys. B688 (2004) 101, hep-ph/0403192

3. A. Vogt, S. Moch and J. Vermaseren, Nucl. Phys. B691 (2004) 129, hep-ph/0404111

4. P. Nogueira, J. Comput. Phys. 105 (1993) 279.

5. J. Vermaseren, math-ph/0010025

6. J. Vermaseren, Nucl. Phys. Proc. Suppl. 116 (2003) 343, hep-ph/0211297

7. S.G. Gorishnii et al., Comput. Phys. Commun. 55 (1989) 381.

8. S.A. Larin, F.V. Tkachev and J. Vermaseren, NIKHEF-H-91-18.

9. S.A. Larin, T. van Ritbergen and J. Vermaseren, Nucl. Phys. B427 (1994) 41.

10. S.A. Larin et al., Nucl. Phys. B492 (1997) 338, hep-ph/9605317

11. A. Retey and J. Vermaseren, Nucl. Phys. B604 (2001) 281, hep-ph/0007294

12. S. Moch and J. Vermaseren, Nucl. Phys. B573 (2000) 853, hep-ph/9912355

13. G. 't Hooft and M. Veltman, Nucl. Phys. B44 (1972) 189.

14. F.V. Tkachov, Phys. Lett. B100 (1981) 65.

15. K.G. Chetyrkin and F.V. Tkachev, Nucl. Phys. B192 (1981) 159.

16. F.V. Tkachov, Theor. Math. Phys. 56 (1983) 866.

17. G. Passarino and M. Veltman, Nucl. Phys. B160 (1979) 151.

18. A. Gonzalez-Arroyo, C. Lopez and F.J. Yndurain, Nucl. Phys. B153 (1979) 161.

19. A. Gonzalez-Arroyo and C. Lopez, Nucl. Phys. B166 (1980) 429.

20. J. Vermaseren, Int. J. Mod. Phys. A14 (1999) 2037, hep-ph/9806280

21. J. Blümlein and S. Kurth, Phys. Rev. D60 (1999) 014018, hep-ph/9810241

22. S. Moch, P. Uwer and S. Weinzierl, J. Math. Phys. 43 (2002) 3363, hep-ph/0110083

23. M.E. Hoffman, J. Algebra 194 (1997) 477.

24. M.E. Hoffman, math.QA/0406589

25. T. van Ritbergen, A.N. Schellekens and J. Vermaseren, Int. J. Mod. Phys. A14 (1999)
41, hep-ph/9802376

26. G. 't Hooft, Nucl. Phys. B61 (1973) 455.

27. W.A. Bardeen et al., Phys. Rev. D18 (1978) 3998.

28. J. Blümlein, (2004), hep-ph/0407044

29. A. Goncharov, Math. Res. Lett. 5 (1998) 497, (available at http://www.math.uiuc.edu/K-theory/0297).

30. J.M. Borwein et al., math.CA/9910045

31. E. Remiddi and J. Vermaseren, Int. J. Mod. Phys. A15 (2000) 725, hep-ph/9905237.

32. S. Catani and F. Hautmann, Nucl. Phys. B427 (1994) 475, hep-ph/9405388

33. V.S. Fadin and L.N. Lipatov, Phys. Lett. B429 (1998) 127, hep-ph/9802290

34. R. Kirschner and L.N. Lipatov, Nucl. Phys. B213 (1983) 122.

35. J. Blümlein and A. Vogt, Phys. Lett. B370 (1996) 149, hep-ph/9510410

36. J.A. Gracey, Phys. Lett. B322 (1994) 141, hep-ph/9401214

37. J.F. Bennett and J.A. Gracey, Nucl. Phys. B517 (1998) 241, hep-ph/9710364

38. G.P. Korchemsky, Mod. Phys. Lett. A4 (1989) 1257.

39. C.F. Berger, Phys. Rev. D66 (2002) 116002, hep-ph/0209107

40. E.A. Kuraev, L.N. Lipatov and V.S. Fadin, Sov. Phys. JETP 45 (1977) 199.

41. I.I. Balitsky and L.N. Lipatov, Sov. J. Nucl. Phys. 28 (1978) 822.

42. T. Jaroszewicz, Phys. Lett. B116 (1982) 291.

43. W.L. van Neerven and A. Vogt, Phys. Lett. B490 (2000) 111, hep-ph/0007362

44. W.E. Caswell, Phys. Rev. Lett. 33 (1974) 244.

45. D.R.T. Jones, Nucl. Phys. B75 (1974) 531.

46. O.V. Tarasov, A.A. Vladimirov and A.Y. Zharkov. Phys. Lett. 93B (1980) 429.

47. S.A. Larin and J. Vermaseren, Phys. Lett. B303 (1993) 334, hep-ph/9302208

48. J. Vermaseren, A. Vogt and S. Moch, to appear.

49. S. Moch, J. Vermaseren and A. Vogt, Nucl. Phys. B621 (2002) 413, hep-ph/0110331 